\title{
A Survey on Naming, Name Resolution and Data Routing in Information Centric Networking $(\mathrm{ICN})$
}

\author{
Alcardo Alex Barakabitze, Tan Xiaoheng, Guo Tan \\ College of Communication Engineering, Chongqing University, China
}

\begin{abstract}
Information-Centric Networking (ICN) is set to replace the current internet architecture which is based on end-to-end communication between hosts. The ICN approach to the network of the future has recently been and is being explored by a number of research projects from Asia, Europe and America. This paper provides a review on three Information Centric Networking (ICN) architectures based on objects/contents naming, name resolution and data routing. The review highlights and briefly describes the naming structures, operation of name resolution and data routing processes of DONA, NetInf and PURSUIT. A summary in tabular form and a comparative study of these three architectures is also given in the paper.
\end{abstract}

Keywords: ICN, Naming, Name Resolution and Data Routing of ICN, Networks.

\section{INTRODUCTION}

Information-centric networking (ICN) is the hot research infrastructure which was meant for a host-to-host topic in recent years, with various research initiatives like communication model.The increasing demand of network DONA [1], CCN/NDN [2][10][16] PSIRP/PURSUIT end -users towards accessing and delivering contents with [3][4][8][11][12], 4WARD [13], CONVERGENCE[15], high volume of digital contents like movies from NetInf [9], SAIL[17],COMET [5-7][14]), MobilityFirst YouTube, time-shift televisions, high definition Video on [18] and ANR Connect [19] targeting this emerging Demand (VoD), photos e.t.c is the one which have driven research area with the aim of shifting from the current the shift from the current internet architecture to a new Internet architecture which is built and designed for a host- architectural plan of the future Internet called Information to-host communications model. The Internet architecture Centric Networking(ICN).ICN targets and puts emphasis today has experienced rapid growth in network traffic of to content objects to be cached/stored in the network which most of the traffics are characterized by the content nodes or routers and also to be accessed anywhere retrieval applications running on top of the Internet different from the current architecture where content are architecture. The Internet has grown tremendously with stored and accessed from the hosts like servers[17].End many new applications being deployed in order to fulfill users in ICN are only interested in the content or object the new requirements from the architecture. These itself in steady of a server location. Indeed, ICN requirements includes the needs to support distribution of architecture is meant to enable in-network storage for contents in a scalable manner, transparency to caching contents, decoupling a content sender/publisher applications, security issues, mobility and many more and receiver/subscriber and also to enable multiparty others [21].Many applications like the MoblieIP, which communication through replication [20].

have been deployed to run and serve the needs of the The main purpose is to have a reliable distribution of current Internet requirements adds some complexity and contents through an efficient communication platform and seems to be only the temporal solutions because they try to services provision which is available in dedicated systems run and match on the architecture which is built on top of like proprietary content distribution networks and peer-to a host-to-host communication model [20][22].The peer (P2P) overlays. The concept behind ICN is to develop contents or objects delivery solutions today from a network which will interprets, processes, and delivers producers or publishers to subscribers/consumer involves contents/information/objects automatically and technologies like Content Delivery Networks (CDNs), independently of its physical location [23]. ICN is to peer-to-peer networks and other players such as CDN replace the host addresses with content names which will providers, Internet Service Providers(ISPs) and Content be exchanged upon user requests or demands and network Providers. However, the current internet content delivery components like routers will be equipped with storage today suffers from heterogeneity problems because its capabilities. Since the content names will be decoupled evolution and deployment to the current Internet from host addresses, then, it will remove the role of the architecture has been triggered by the market needs rather current IP address which plays as an identifier and a than the coherent internet architectural plan. In addition to locator. By directly naming contents, it will enable inthat, the caching solutions proposed in the current internet network caching (routers to store contents) which will also infrastructure have some inefficiency since they are results in the improved delivery of popular contents in the matching the content centric problem to a network

Internet. In addition, each content will now be 
authenticated and identified uniquely with no any network approaches as shown in Table 1 which stipulate a number host associations [29]. of research projects with the links to their official The key functionalities and general features in all ICN websites. This attention is also verified by the recent architectures are naming, Name resolution and data establishment of the Information-Centric Networking routing, mobility, Caching and Security [24-28].

Research Group (ICNRG) http://irtf.org/icnrg) within the The future Internet architecture (ICN) has drawn attention Internet Research Task Force (IRTF) [28][48]. However, to the Internet research community and the research these ICN project are still in an ongoing process to community has made an increasing interest in the ICN develop the future Internet architecture.

Table 1: Major ICN Research Projects for the Future Internet Architecture.

\begin{tabular}{|l|l|l|l|}
\hline Project & Website & Project Sponsor & Time Frame \\
\hline TRIAD[49] & http://gregorio.stanford.edu/triad/ & United States of America & Jul 1999-Dec 2002 \\
\hline DONA[1] & http://www.sigcomm.org/node/2633 & $\begin{array}{l}\text { University Califonia } \\
\text { Berkeley }\end{array}$ & No data collected \\
\hline AsiaFI & http://www.asiafi.net/ & Asia & Sept 2007- \\
\hline COMET[14] & http://www.comet-project.org/ & Europe & Jan 2010-Dec 2012 \\
\hline $\begin{array}{l}\text { ANR } \\
\text { Connect[19] }\end{array}$ & http://www.anr-connect.org/ & French Funded Project & Jan 2011- Dec 2012 \\
\hline Convergence[15] & http://www.ict-convergence.eu/ & Europe & Jun 2010-Feb 2013 \\
\hline GreenICN[51] & http://www.greenicn.org/ & Europe & $\begin{array}{l}\text { Apr 2013 - Mar } \\
2016\end{array}$ \\
\hline NDN[16] & http://www.named-data.net/ & United States of America & $\begin{array}{l}\text { Sep 2010- Aug } \\
2013\end{array}$ \\
\hline NetInf[50] & http://www.netinf.org/ & NSA-FA Program & Jan 2008 - Jun2010 \\
\hline PSIRP[11] & http://www.psirp.org/ & Europe & Jan 2008-Jun2010 \\
\hline PURSUIT[12] & http://www.fp7-pursuit.eu/ & Europe & Sep 2010-Feb 2013 \\
\hline 4WARD[13] & http://www.4ward-project.eu/ & Europe & Jan 2008-Jun 2010 \\
\hline SAIL[17] & http://www.sail-project.eu & Europe & Aug 2010-Jan 2013 \\
\hline MobilityFirst[18] & http://mobilityfirst.winlab.rutgers.ed & NSA-FIA Program & Sep 2010-Sep 2013 \\
\hline
\end{tabular}

This paper illustrates three ICN architectures namely returned address to establish the end-to-end DONA, NetInf and PURSUIT with focusing only in three communication session. This is a location dependent ICN features which are Naming, Name Resolution and mainly on IP address which faces problems to archiving Data Routing. The rest of this paper is organized as the persistence and services availability requirements. In follows: Section 2 provides a brief description of the this manner, three features under naming in ICN have naming, name resolution and data routing in ICN. Section been proposed [28] which are name-data integrity that 3- 5 gives in depth explanations of the mentioned ICN establish a verifiable binding between the object and its architectures. Section 6 summarizes the highlighted parts name; object authenticity which ensures authentication of of the descriptions by giving a comparative study of the received objects to a receiver in such a way that the three ICN architectures. Then section 7 concludes the received object represent the actual object published on paper.

\section{NAMING, NAME RESOLUTION AND DATA ROUTING OF ICN}

\section{$2.1 \quad$ Naming in ICN} the network and the object's provenance which enables to know who published the object on the network.

The proposed naming mechanisms of ICN architectures are location independent. These proposed naming schemes in ICN are:

The main abstraction of ICN is the Named Data Object (NDO) which includes webpages, videos, photos, documents, live streaming and interactive media [28].One of the ICN feature is Naming. Naming data objects is an important aspect of ICN. Since names are used to indentify NDO independent of its location then, ICN needs unique names for every NDO [28].The IP address in the current internet architecture performs a key role between parts in communication.The current end-to-end communication paradigm performs two separate sessions in the process of establishing a communication. The first step is to resolve the name to an IP address and then as a separate session, send a communication request using the

- Hierarchical name space-Which has a similar structure like the URL.

Advantages

a) It enables routing information aggregation.

b) The routing system is improved in terms of scalability.

- Flat and Self-certifying namespace:-means that the name-data integrity is to be verified with no need of a public key infrastructure (PKI)[28]. Flat naming offers uniqueness and persistency.

In general, naming mechanisms in different ICN designs may range from flat to hierarchical and sometimes other approaches employ the human readable naming scheme 
which enables a user to type the names manually and retrieve contents according to his or her needs [21].

2.2 Name Resolution, Data Routing and Forwarding in ICN. ICN manages the process of routing and forwarding NDO packets through two different steps namely the name resolution which is a 2-way mechanism and the Name Based Routing (NBR) which is just a 1-way mechanism [30].

The Name Resolution step enables a subscriber/customer to search for NDO by using names. The first step is to map the name and the source locators where the NDO is stored [31], the second step is to forward the request message from the subscriber to the source. However, this 2-step mechanism is called the Name Resolution System (NRS) for providing translation. One of the drawbacks of this mechanism is that, the NRS itself become a point-offailure which can results into many of the NDO published and registered on that NRS to be unavailable[31].The only advantage of this approach is that, it ensures subscribers to find the requested NDO because NRS are providing the pointer to the NDO source.

Name-Based or Data Routing is a 1-way mechanism where the NDO request is forwarded by content routers (CR) and the CR decides locally the next hop of the NDO request based on NDO name[31].According to Choi [32],there are two types of routing models in ICN design approaches which are:

a) Unstructured Routing Model that works similar to the current IP routing but with some modifications.

b) Structured Routing Model which utilizes the Distributed Hash Table (DHT) to provide a lookup and routing service [31][32].

In principle, name resolution is the process of mapping or matching a content name to a publisher/provider/source which can then provide the requested content. Data routing is the process of making a required path for transferring the content/information from a publisher up to the subscriber/customer.

The name resolution and data routing in ICN design can be integrated together to form a coupled approach where the requested content by a subscriber is routed to content providers and then this provider sends the content back to the subscriber by using the reverse path over which the request was forwarded[21]. When the two functions are not integrated together, it form the decoupled approach where there are no restrictions on the path that will be used to forward data to and from a subscriber to the content publisher/provider [21].The ICN design models for the future internet architecture available so far have proposed different naming system as well as different data routing processes. Some ICN architectures perform name resolution and data routing separately in such a way that they perform name resolution first and then perform data routing. Other ICN proposals tried to combine these transactions together. The following section provides in depth description of the naming, name resolution and data routing process of DONA, PURSUIT and NetInf ICN architectures

\section{DATA ORIENTED NETWORK ARCHITECTURE (DONA) NAMING}

DONA [1] proposes to use names which are flat, application-independent, location-independent ,globally unique[21] and a self certifying naming scheme with a resolution infrastructure that is organized in a hierarchical manner which intend to achieve three basic objectives namely: a reduced users' request latency, persistence and provenance of contents [30].Every contents or objects in DONA have an association with a principal and all names have the form P:L where $\mathrm{P}$ represents the cryptographic hash of the owner's public key[21] and L represents a label that identifies uniquely the contents with respect to the principal P[30].

An example of naming scheme in DONA is shown below

FileType <String>: $\boldsymbol{d o c x}$

Paper title $<$ String $>$ : Review of Naming in ICN

Author <ListofString >: Alcardo Alex

Institution $<$ String >: Chongqing University

Year <Integer>: 2014

3.1 Name resolution and data routing

The Resolution Handlers (RHs) which are the specialized servers performs name resolution in DONA. Every Autonomous System (AS) has more than one logical RHs which are connected to each other forming a hierarchical name resolution service[21][28].The inter-domain routing solutions is built together with the hierarchical resolution systems so as to enable the name resolution and data routing through the data routing policies established between two or more ASs.

Figure 1 below shows the mechanism of data routing and name resolution in DONA.

The publisher or principal or owners of content is responsible to publish the NDOs into the network where nodes that are authorized to provide contents or information have to register to the resolution infrastructure which consists of RHs.

The publisher or principal sends a

REGISTER message with the content's name to its local $\mathrm{RH}$, the $\mathrm{RH}$ stores the pointer to the principal $\mathrm{P}$ as shown by arrow 1 .The local $\mathrm{RH}$ of the principal then sends information of this registration to other RHs which are in the same domain through their established data routing policies in the network (see arrow 2-3). After receiving the registration sent by the local RH of the principal, each peering $\mathrm{RH}$ then stores a mapping of object's names and the address of the local $\mathrm{RH}$ which were forwarded by the publisher during registration.

This registration continues up to tier- 1 providers which enables RHs in tier-1 to become aware of this publisher registration throughout the whole network. On the subscriber part, a FIND message is sent to its local RHs which forwards the request to RHs peers to find a content matching as shown in arrow 4 to 5.The pointers created while publishing a registration from a principal are used to reach the publisher of the content as shown in arrow 6 and 7.This process becomes successful if the requested content is already published in the network. 


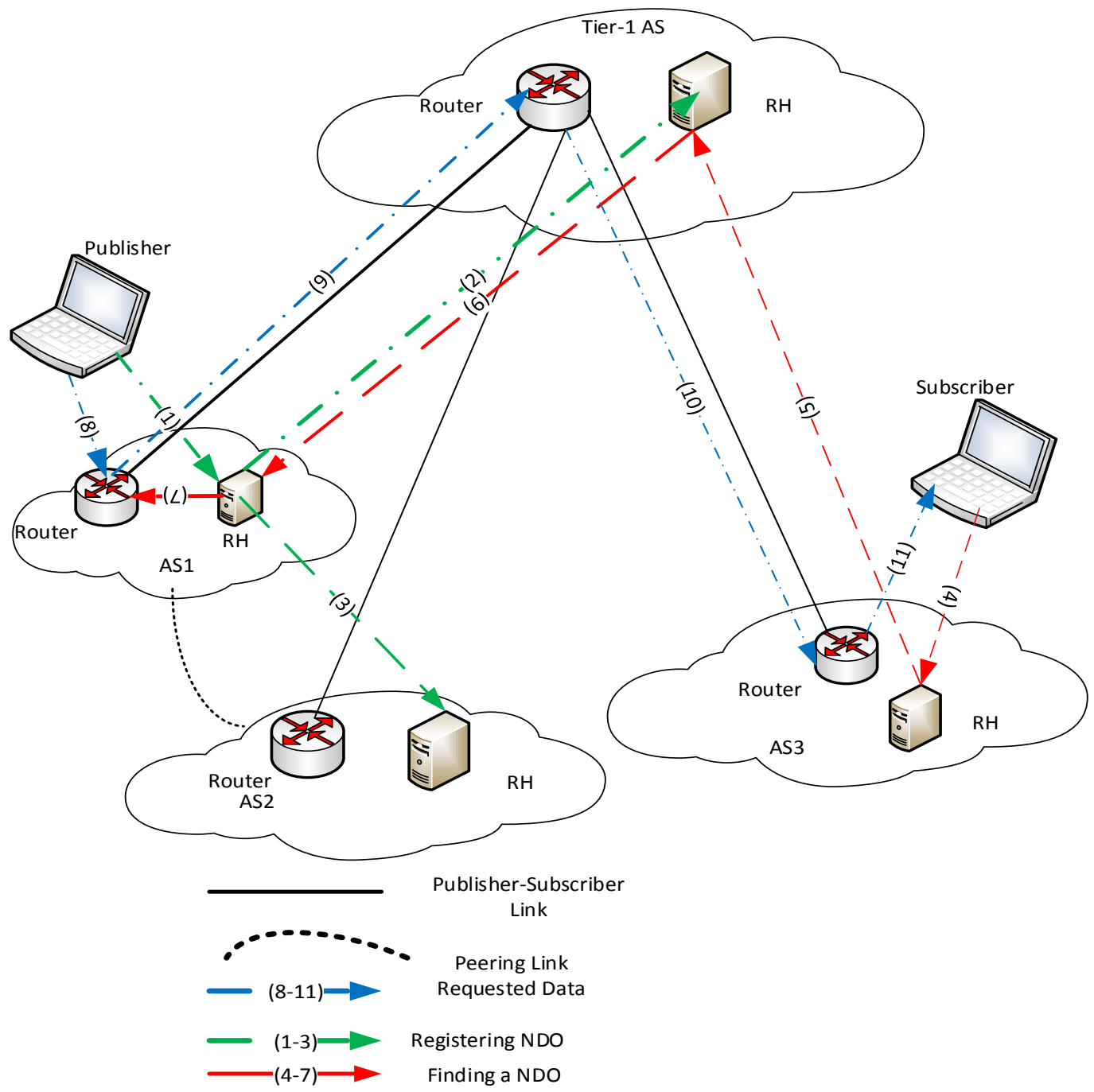

Figure 1: The DONA architecture.

However, subscribers/customers may request a wildcard Multicast channels can be supported in DONA which is a FIND messages to ask for unchangeable data with a mechanism that enables resolution handlers to cache the specific label, regardless of its purveyor [21][28][30].

3.2 Data routing:

DONA takes two forms of data routing which are decoupled and coupled data routing. In the decoupled data routing, the traditional IP routing and forwarding mechanisms can be used to send back the requested content to a subscriber after a content match from the publisher. This requires the normal transmission schemes of network traffic and the established routing policies between the subscribers' ASs and publisher's AS's.

In the coupled data routing model, the FIND message from a subscriber, records every RH and the routes it traverses together with its associated sequences of ASs that the request took to reach a publisher of the content in the network.

When a content match occurs to the publisher, the routes recorded in the FIND message will be used to forward the content back to the subscriber as shown in arrows 8-11.
FIND message for duration of time until the message expires [21]. In this case, if another find message arrives to the RH looking for the same message, then the RH merges these two messages into a single entry but with multiple path labels for the responses [21]. In this manner, the multicast distribution tree is created and hence the multicast channels.

\section{PUBLISH SUBSCRIBE INTERNET TECHNOLOGY (PURSUIT)}

The PURSUIT ICN design for the future internet architecture is the continuation of the Publish Subscribe Internet Routing Paradigm (PSIRP)[11] which are both funded by the EU Framework 7 Project[21][31].The PUSRSUIT project have implemented the structure of the publish-subscribe protocol stack which will replace the traditional IP protocol stack in the current internet architecture. The PURSUIT ICN architecture consists of three main functions as shown in figure 1. 


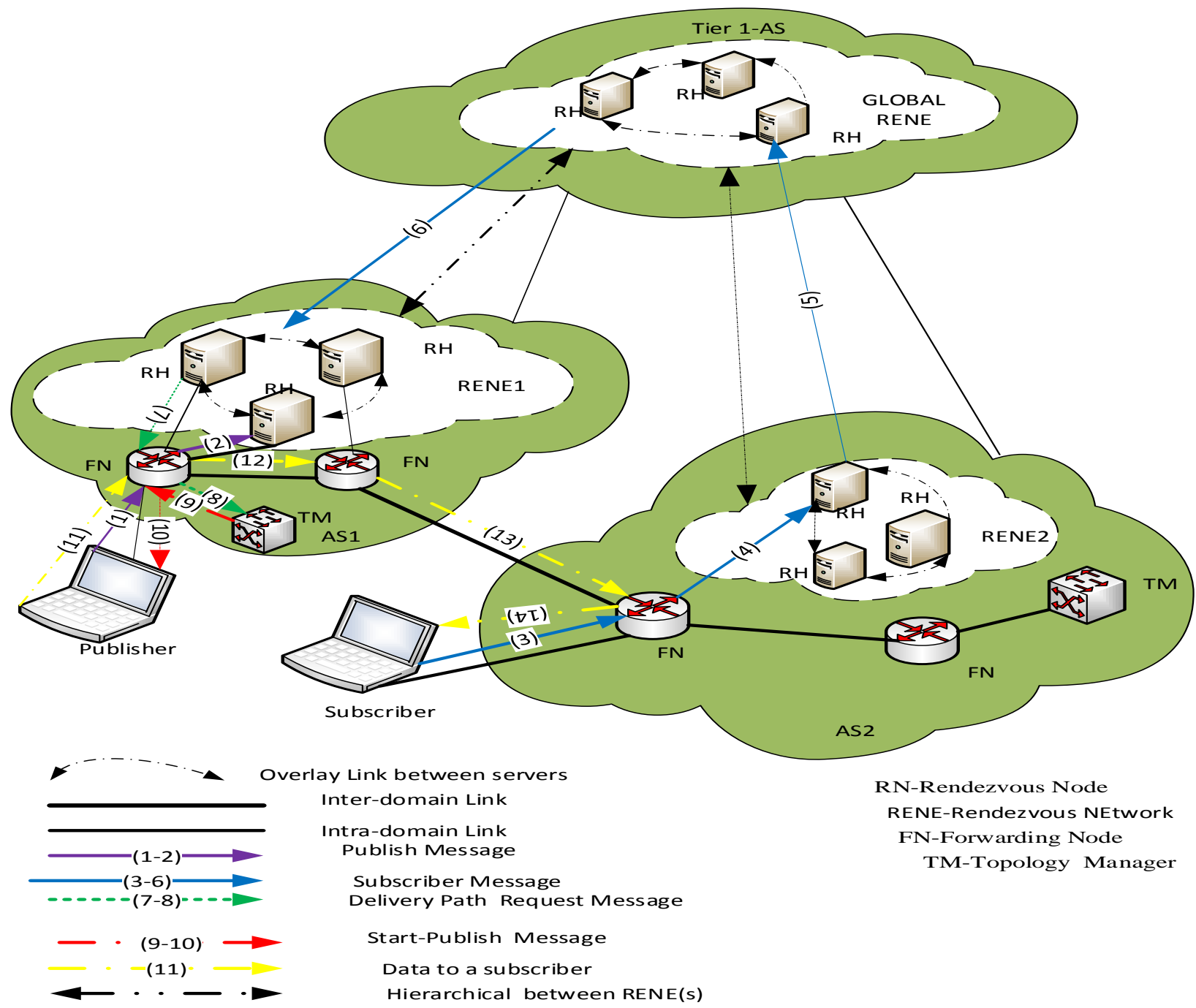

Figure 2: The PURSUIT architecture [21].

The three core parts of PURSUIT are:-

- $\quad$ Rendezvous Function(RF)

- Topology Management Function(TMF)

- Data Forwarding Function (DFF).

a) The Rendezvous Function

The Rendezvous function which operates at the Rendezvous Node (RN) is the most important part in the PURSUIT ICN model since it establishes a connection between the subscriber and the publisher for an information item on the network infrastructure. It also initializes the information item delivery from the publisher to the subscriber by directing the topology management function to create a possible route for forwarding data to a subscriber [31].

b) The Topology Management Function(TMF)

The TMF is responsible for creating a routing policy and also to collect the topology information of its domain. It also performs the exchange of routing information with other topology management peers so as to enhance routing of information globally. The Topology Manager (TM) operates the TMF and one network domain has one TM as shown in figure 2 [21].

c) The Data Forwarding Function(DFF)

The DFF is performed on the Forwarding Node (FN) which is responsible for directing the information item to the subscriber who requested particular information. In the PURSUIT ICN design the FN has capabilities also of caching or storing an information item.

\section{$4.1 \quad$ Naming}

The NDO in PARSUIT are called information items [31].These information items are indentified by a unique pair of identifiers called the Rendezvous ID (Rid) and the Scope ID (SId). SId is responsible to keep items of related information together and the Rid is responsible to keep and identify information of a particular item [33]. As shown in figure 3 , every information item must be in at least one scope. The scopes are responsible to provide:- Policy and boundaries enforcement to an information item [31].

- Access right for each group of information items [31]. 


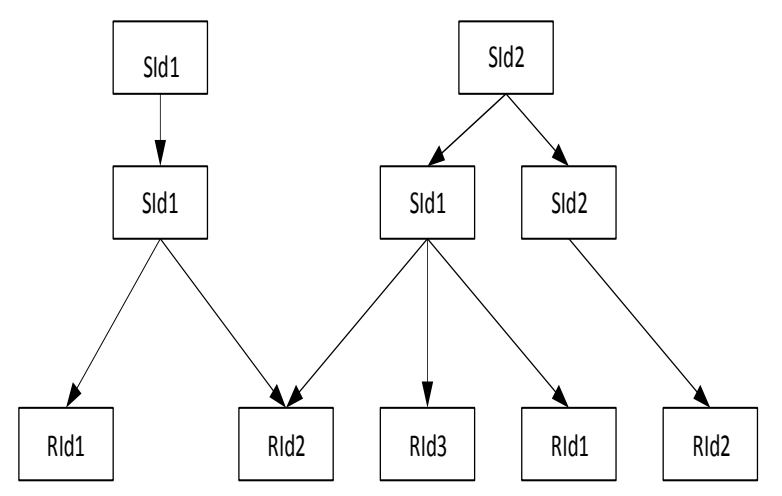

Figure 3: Information item model of PURSUIT [11][31] PURSUIT utilizes the flat naming scheme which has sequences of SIds and an RId. As shown in the PURSUIT information item model (see figure 3), a name of an information item takes a path from the root of the tree to the leaf node which actually is the RId. However, multiple names of an information item can be used to specify the same piece of an information item. i.e SId2/SId1/Rid2 or SId1/SId1/Rid2 can be used to specify Rid2.

4.2 Name Resolution and Data Routing.

In PURSUIT, the name resolution and data routing is implemented by a collection of Rendezvous Nodes (RNs), the Rendezvous Network (RENE), Topology Manager (TM) and the Forwarding Nodes (FNs). According to [34] and [35],the RENE in PURSUIT, is designed and implemented as a hierarchical Distributed Hash Table(DHT).As shown in figure 2, the global RENE in tier-1 connects together the RENE1 in AS1 and RENE2 in AS2.

From figure 2 above, A publisher publishes information item of identifiers (SId, RId) to its local RN which is the owner of the scope as shown in arrows 1-2. The owner of the scope is not necessarily to be in the same domain and therefore can also be a source RNs from other domains such as RN from RENE2 in AS2.The subscriber who is need of the same item specifies RId and SId in order to request for an information item and sends out a subscription message through its local $\mathrm{RN}$ towards the scope owner RN using RId as shown in arrow 3-6.

The Topology Manager (TM) node in RENE 1 is then instructed by the RN to create a route that will connect the publisher and the subscriber for data delivery as shown in arrows 7-8.The START-PUBLISH route as a message is sent to the publisher by the TM which will be utilized in sending the information items through a set of Forwarding Nodes (FNs). The START-PUBLISH route is shown in arrow 9-10. The information item which is forwarded via FNs, uses the Bloom Filter's Forwarding Identifier (FId) to decide where to send the packet [36].

In PURSUIT, the RENE performs the process of resolving names or name resolution while the TMs perform the data routing process which is executed by the FNs [21].

\section{NETWORK OF INFORMATION (NETINF)}

Network of Information (NetInf) is a part of the EU FP7 projects 4WARD and SAIL[50]. The NetInf is one of three "Scalable and Adaptive Internet Solutions" approaches in the SAIL project of which the other ones are Open Connectivity Services [37] and Cloud Networking [38].

The NetInf project is mainly based on three core functionalities:

a) The idea of unique naming of information objects without imposing a hierarchical naming structure like the approaches used in 4WARD and DONA [39].

b) Receiver-oriented transport as in $\mathrm{CCN}$.

c) A multi-technology or multi-domain approach than can leverage different underlying network services and employ different name resolution/name-based routing and transport mechanisms [39].

\subsection{Naming in NetInf}

The naming in NetInf takes a flat-ish [21] structure which enables a certain degree of flexibility similar to resource identification in the current web today. The NetInf naming therefore includes some Uniform Resource Identifier (URI) concepts and have developed the Named Information (ni:) URI scheme that is specified by Stephen et.al [40] and that of Philip et.al [41] for Internet drafts. As shown in figure 4 below, the naming system proposed by NetInf is a flat and self-certifying like in DONA and it consists of two parts which are A: L, where A is the hash of the owner's public key and $\mathrm{L}$ is a label chosen by the owner. This public-private key pair is taken as a binding to the content or an information object.

\begin{tabular}{|l|l|l|}
\hline Type & $\mathrm{A}=\mathrm{Hash}\left(\mathrm{PK}_{\mathrm{IO}}\right)$ & $\mathrm{L}=\{$ attributes \\
\hline
\end{tabular}

Figure 4: The basic naming structure of NetInf [42]

Every part in the structure can be considered as a hash which then allows for self-certification. In addition to that it also allows different type of a string data type therefore allowing the normal Uniform Resource Locators (URLs)[39]. The NetInf requires that, a subscription have to match with the publication only if the actual name on the network is found to be similar with the matching pair between that of the subscriber and a publisher otherwise an information object will seem to be missing. In case of routing, names in NetInf can be hierarchical where routers can determine how to route an information object to a subscriber by using the longest prefix matching mechanism similar to the one used in NDN [43].

5.2 Name Resolution and Data routing in NetInf. The Name Resolution function is the main part of NetInf ICN architecture which utilizes the Name Resolution System (NRS) to provide resolution services to NetInf subscribers. The NRS can play also a function of a namebased routing system [39]. A specific NRS in [44] has been designed based on the Resolution EXchange (REX) sub-system of Multilevel Distributed Hash Table (MDHT).This specific NRS integrate the name-based forwarding system in NetInf and the name resolution function. It is used to show how the resolution process can be performed over a global network [39].

Requests and response messages from subscribers are forwarded and resolved for an information objects in NetInf design architecture. As in the current Internet architecture which has multiple routing protocols, different 
parts of the NetInf network may also have many different integrating the name-based routing and the aspects of the requirements and therefore needing also multiple different name resolution, the NetInf can be able therefore to use a routing protocols. In this manner, the NetInf ICN hybrid request routing or forwarding scheme. The architectural design can be able to support different integration of name resolution and name-based routing can request or response and routing or forwarding protocols also support Late Name Binding (LNB) which is a strategy like the Open Shortest Path First (OSPF) for local domains that enables the name resolution process to be done at a [43]. The design and implementation principles in NetInf node which is near to the current area of a moving therefore enable an easy way of plugging a new name host[21][43][28]. resolution, forwarding or even a routing protocol. By

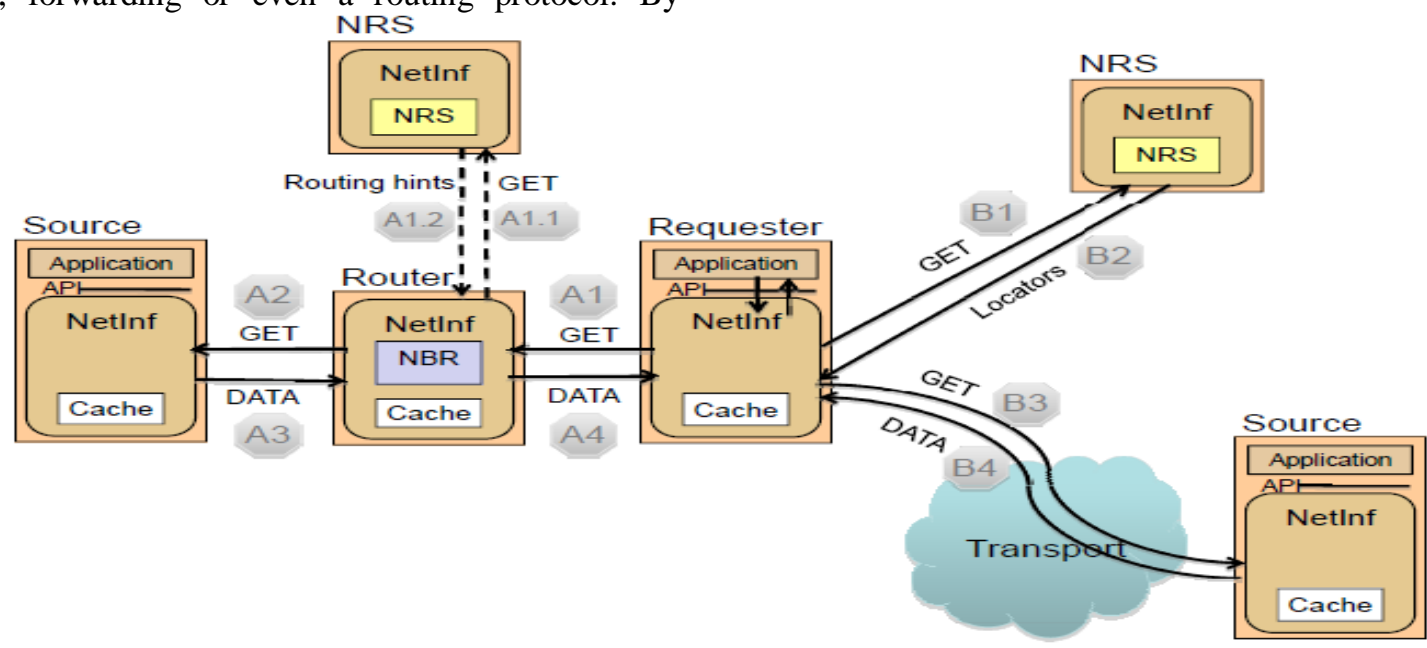

Figure 5: Final NetInf Architecture and message flow [43]

The NetInf ICN design can easily adapt to different similarities as well some differences in the naming, name network environments because of its capability to retrieve Resolution and the way data is routed from the client to the information objects through a name resolution, name- the source. Table 2 shows a comparison of the three ICN based routing as well as a hybrid operation[21][28].In design for the future Internet architecture

the case of name resolution, the publisher/source is DONA has a flat naming scheme structure which consists responsible to publish the NDOs through a registration of of a principal and a label part. The names in DONA are the name/locator binding to a NRS while in the case of not human readable and the granularity of NDO is the name-based routing, the publisher/source is responsible to object itself. The name resolution is managed by the RHs publish the NDOs by announcing routing information in a which are organized by the Autonomous System (AS) routing protocol [43]. Figure 5 shows an example of hierarchy [21].The name-based routing via RHs handles name-based routing, name resolution and the hybrid all NDO requests while the routing of NDO back to the operation in NetInf. The requester through a GET message client follows the reverse path of the request.

resolve the NDO into a set of routing hints and can be able to query NRS. Subsequently, the routing hints are used to retrieve an information object from available sources using the underlying transport network such as the IPv4 network as shown in steps B1-B4. Alternatively to that, the requester can sends a GET request hop-by-hop between NetInf routers/caches until a copy of an information object is found through the name-based routing as shown in steps A1-A4.

Steps A1.1-A1.2 shows a hybrid operation which occurs when the router for example in step A2 does not have sufficient information to perform name-based routing. In this case, the NRS therefore returns routing hints which are partial locators that can direct a GET message in one or more routes where more information about the requested information object may be found [21].

\section{SUMMARY AND COMPARATIVE ANALYSIS} OF THE THREE ICN DESIGN APPROACHES.

The three discussed ICN design approaches have some

NetInf takes a flat-ish name structure. The name resolution and data routing in NetInf can be a coupled, decoupled or hybrid operation. In the coupled case, the NDO request is enabled to accumulate routing state during name resolution. In the decoupled case, the Distributed Hash Table (DHT)-based name resolution is responsible to return the content locator, and in the hybrid case, the name resolution is responsible to return the routing hints in order to assist the coupled operation [21].

The PURSUIT takes a flat naming scheme which is not human readable as in DONA. Different from DONA, the name in PURSUIT consists of scope Id and the rendezvous Id. The NRS is responsible to match the subscriber and a publisher of an information object. The NDO requests are handled by the NRS while the routing of NDO back to the client is handled by the source routes using a techniques which is based on bloom filters [21][36]. 
International Journal of Advanced Research in Computer and Communication Engineering Vol. 3, Issue 10, October 2014

Table 2:Comparison of ICN Designs for the future Internet.

\begin{tabular}{|c|c|c|c|}
\hline $\begin{array}{l}\text { Comparison } \\
\text { Parameter }\end{array}$ & DONA & PURSUIT & NetInf \\
\hline Namespace & $\begin{array}{l}\text { Flat naming scheme } \\
\text { consisting of } P: L \text { parts }\end{array}$ & $\begin{array}{l}\text { Flat naming scheme } \\
\text { consisting of Scope and } \\
\text { Rendezvous parts }\end{array}$ & $\begin{array}{l}\text { Flat naming scheme } \\
\text { consisting of } A: L \text { parts }\end{array}$ \\
\hline Human Readability & NO & NO & NO \\
\hline Name Certification & $\begin{array}{l}\text { Self-certified by a } \\
\text { cryptographic hash }\end{array}$ & $\begin{array}{l}\text { Packet level authentication } \\
\text { can be self -certifying }\end{array}$ & $\begin{array}{l}\text { Self-certified by a } \\
\text { cryptographic hash }\end{array}$ \\
\hline Granularity of NDO & Object & Object & Object \\
\hline $\begin{array}{c}\text { Name Resolution and Data } \\
\text { routing }\end{array}$ & Coupled or Decoupled & Decoupled & Decoupled \\
\hline $\begin{array}{l}\text { Name Resolution is } \\
\text { handled by }\end{array}$ & Resolution Handler(RH) & Rendezvous Node(RN) & $\begin{array}{c}\text { Resolution } \\
\text { Exchange(REX)) }\end{array}$ \\
\hline Data retrieval path & $\begin{array}{l}\text { Reverse Request Path or } \\
\text { a standard IP routing }\end{array}$ & $\begin{array}{c}\text { Follows the route provided } \\
\text { by the Topology } \\
\text { Manager(TM) }\end{array}$ & $\begin{array}{l}\text { Reverse Request Path or } \\
\text { Standard IP routing }\end{array}$ \\
\hline $\begin{array}{l}\text { Inter-domain } \\
\text { Communication }\end{array}$ & $\begin{array}{c}\text { AS level RH hierarchy for } \\
\text { name resolution }\end{array}$ & $\begin{array}{l}\text { Hierarchical DHT based } \\
\text { Interconnection overlay } \\
\text { solution }\end{array}$ & MDHT \\
\hline
\end{tabular}

\section{CONCLUSION}

This paper survey has explored the three promising ICN architecture design for the future Internet architecture which are DONA, NetInf, PURSUIT. We have described in more detail the operations of these ICN architecture regarding to naming, name resolution and data routing which are among the features of every ICN architecture. Along with this, the review has provided a comparative analysis of the three ICN designs. Although there is a need of migrating from the current Internet architecture to the new ICN Internet architecture but still there a lot of challenges which are related to developing efficient scalable routing schemes, congestion control mechanisms, Quality of service (QoS) approaches, and efficient caching decision policies, security and privacy issues, new ICN application-protocols design, new business models between different actors or players on the Internet as well as new legal and regulatory frameworks [48].We argue that, ICN is a new paradigm with an intention to replace the current Internet architecture which was meant for communication between hosts which does not suits with the high demanding needs of users today. In this regard, there is a high need to research more on the outlined challenges so as to meet the future requirements of the ever growing number of Internet users who are interested in the contents regardless of their location on the network.

\section{ACKNOWLEDGMENT}

We would like to convey our gratitude to the entire community of Chongqing University for creating a unique and peaceful learning environment. The direct and indirect support we received in accomplishing this paper from academic staff in the college of Commutation Engineering is highly appreciated

\section{REFERENCES}

[1] T. Koponen, M. Chawla, B. Chun, A. Ermolinskiy, K. Kim, S. Shenker, I. Stoica, A data-oriented (and beyond) network architecture, ACM SIGCOMM Computer Communication Review, vol. 37, ACM, 2007, pp. 181-192.

[2] V. Jacobson, D. Smetters, J. Thornton, M. Plass, N. Briggs, R. Braynard, Networking named content, in: Proceedings of the 5th International Conference on Emerging Networking Experiments and Technologies, ACM, 2009, pp. 1-12.

[3] D. Trossen et al., Conceptual Architecture: Principles, Patterns and Subcomponents Descriptions, May 2011. <http://www.fp7pursuit.eu/ PursuitWeb/>.

[4] P. Jokela et al., LIPSIN: Line speeds publish/subscribe internetworking, in: Proc. ACM SIGCOMM, Barcelona, Spain, 2009.

[5] G. Garcia et al., COMET: Content mediator architecture for content-aware networks, in: Proc. of the Future Network and Mobile Summit 2011, Warsaw, Poland, IEEE, June 2011.

[6] G. Pavlou, N. Wang, W.K. Chai, I. Psaras, Internet-scale Content Mediation in Information-centric Networks, Ann. Telecommun., Special Issue on Networked Digital Media, Springer, in press. http://dx.doi.org/10.1007/s12243-012-0333-8. 
[7] W.K. Chai et al., CURLING: Content-ubiquitous resolution and [33] delivery infrastructure for next-generation services, IEEE Commun. Mag. 49 (3) (2011) 112-120.

[8] D. Lagutin, K. Visala, S. Tarkoma, Publish/subscribe for internet: [34] Psirp perspective, Towards the Future Internet Emerging Trends from European Research 4 (2010) 75-84.

[9] B. Ahlgren, M. D’Ambrosio, M. Marchisio, I. Marsh, C. Dannewitz, B Ohlman, K. Pentikousis, O. Strandberg, R. Rembarz, V. Vercellone, Design considerations for a network of information, in: Proceedings of the 2008 ACM CoNEXT Conference, ACM, 2008, p. 66

[10] L. Zhang, D. Estrin, J. Burke, V. Jacobson, J. Thornton, D. Smetters, [36] B. Zhang, G. Tsudik, D. Massey, C. Papadopoulos, et al., Named Data Networking (NDN) Project, Tech. Rep., PARC, Tech. Report ndn0001,2010

[11] FP7 PSIRP project. [Online]. Available: http://www.psirp.org/

[12] FP7 PURSUIT project. [Online]. Available: http://www.fp7pursuit.eu/PursuitWeb/

[13] FP7 4WARD project. [Online]. Available: http://www.4wardproject.eu/

[14] FP7 COMET project. [Online]. Available: http://www.cometproject.org/

[15] FP7 CONVERGENCE project. [Online]. Available: http://www.ictconvergence.eu/

[16] NSF Named Data Networking project. [Online]. Available: http://www.named-data.net/

[17] FP7 SAIL project. [Online]. Available: http://www.sail-project.eu/

[18] NSF Mobility First project. [Online]. Available: http://mobilityfirst.winlab.rutgers.edu/.

[19] ANR Connect project. [Online]. Available: http://anr-connect.org/

20] Bengt Ahlgren, Christian Dannewitz, Claudio Imbrenda, Dirk Kutscher, and Börje Ohlman .A Survey of Information-Centric Networking, IEEE Communications Magazine • July 2012

[21] George Xylomenos, Christopher N. Ververidis, Vasilios A. Siris, Nikos Fotiou, Christos Tsilopoulos, Xenofon Vasilakos, Konstantinos V. Katsaros, and George C. Polyzos, A Survey of Information-Centric Networking Research, IEEE COMMUNICATIONS SURVEYS \& TUTORIALS,September 2013.

[22] M. Handley, "Why the Internet only just works," BT Technology J.vol 24, no. 3, pp. 119-129, July 2006.

[23] G. Carofiglio , G. Morabito, L. Muscariello, I. Solis, M. Varvello, From content delivery today to information centric networking, Computer Networks 57 (2013) 3116-3127,July 2013.

[24] A. Ghodsi, T. Koponen, J. Rajahalme, P. Sarolahti, and S. Shenker, "Naming in content-oriented architectures," in ACM Workshop on Information-Centric Networking (ICN), 2011.

[25] P. Stuckmann and R. Zimmermann, "European research on future [46] Internet design," IEEE Wireless Commun., vol. 16, no. 5, pp. 14-22, October 2009.

[26] J. Pan, S. Paul, and R. Jain, "A survey of the research on future Internet architectures," IEEE Commun. Mag., vol. 49, no. 7, pp. 2636, July 2011

[27] J. Choi, J. Han, E. Cho, T. Kwon, and Y. Choi, "Survey on content [48] oriented networking for efficient content delivery," IEEE Commun. Mag., vol. 49, no. 3, pp. 121-127, March 2011

[28] B. Ahlgren, C. Dannewitz, C. Imbrenda, D. Kutscher, and B. Ohlman, "A survey of information-centric networking," IEEE Commun. Mag., vol. 50, no. 7, pp. 26-36, July 2012.

[29] Wei Koong Chai, Diliang He, Ioannis Psaras, George Pavlou.Cache "less for more", in information-centric networks (extended version), Computer Communications 36 (2013) 758-770, www.elsevier.com/ locate/comcom

[30] M.F. Bari; S. Chowdhury; R. Ahmed; R. Boutaba; B. Mathieu, A survey of Naming and Routing in Information Centric Networks,â€• IEEE Comm Mag, vol. 50, no. 12, 2012, pp. 44-53. http://ieeexplore.ieee.org/stamp/stamp.jsp?tp=\&arnumber=6384450\&i snumber $=6384439$.

[31] Dolvara Gunatilaka, Recent Information-Centric Networking Approaches,[Online] available at http://www.cse.wustl.edu/ jain/cse570-13/ftp/icn/

[32] J. Choi; J. Han; E. Cho; T. Kwon; Y. Choi, â€œA Survey on ContentOriented Networking For Efficient Content Delivery,â€• IEEE Comm Mag, vol. 49, no. 3, 2011, pp.121-127. http://ieeexplore.ieee.org/stamp/stamp.jsp?tp=\&arnumber=5723809\&i snumber $=5723785$
D. Trossen and G. Parisis, "Designing and realizing an informationcentric Internet," IEEE Commun. Mag., vol. 50, no. 7, pp. 60-67, July

J. Rajahalme, M. S“arel“a, K. Visala, and J. Riihij”arvi, "On namebased inter-domain routing," Computer Networks, vol. 55, no. 4, pp. 975-986, March 2011 .

K. V. Katsaros, N. Fotiou, X. Vasilakos, C. N. Ververidis, C. Tsilopoulos, G. Xylomenos, and G. C. Polyzos, "On inter-domain name resolution for information-centric networks," in Proc. IFIPTC6 Networking Conference.2012.

36] B. H. Bloom, "Space/time trade-offs in hash coding with allowable errors," Communications of the ACM, vol. 13, no. 7, pp. 422-426, July 1970.

[37] SAIL Project. Architectural Concepts of Connectivity Services. Deliverable FP7-ICT-2009-5- 257448-SAIL/D.C.1, SAIL EU FP7 project, July 2011. Available online from http://www.sailproject.eu.

38] SAIL Project. Cloud Networking Architecture Description. Deliverable FP7-ICT-2009-5- 257448-SAIL/D.D.1, SAIL EU FP7 project, July 2011. Available online from http://www.sailproject. eu.

[39] SAIL-(D-B.1).The Network of Information:

Architecture and Applications: Objective FP7-ICT-2009-5-257448/D-

3.1, available at http://www.sailproject.eu/wpcontent/uploads/2011/08/SAIL_DB1_ v1 0 final-Public.pdf

[40] Stephen Farrell, Dirk Kutscher, Christian Dannewitz, Boerje Ohlman, and Phillip Hallam-Baker. The Named Information (ni) URI Scheme: Core Syntax. Internet Draft draft-farrelldecadeni-00, Work in progress, October 2011

[41] Phillip Hallam-Baker, Rob Stradling, Stephen Farrell, Dirk Kutscher, and Boerje Ohlman.The Named Information (ni) URI Scheme: Parameters. Draft-hallambaker-decade-ni-params-00, Work in progress, October 2011.

42] Christian Dannewitz, Jovan Goli'c, B“orje Ohlman, and Bengt Ahlgren, "Secure Naming for a Network of Information", IEEE INFOCOM 2010

[43] SAIL Project. (2013, January) SAIL deliverable B.3 (3.3): Final NetInf architecture. [Online]. Available: http://www.sailproject. Eu/deliverables/.

[44] M. D'Ambrosio, C. Dannewitz, H. Karl, and V. Vercellone. MDHT: A Hierarchical NameResolution Service for Informationcentric Networks. In ACM SIGCOMM Workshop on InformationCentric Networking (ICN 2011), Ottawa, Canada, August 2011.

] Content Centric Networking project. [Online]. Available: http://www.cenx.org/

V. Jacobson, "A new way to look at networking," Google Tech Talk, August 2006

[47] Huichen Dai, Jianyuan Lu, Yi Wang, Bin Liu(2012), A Two-layer Intra-domain Routing Scheme for Named Data Networking, Globecom 2012 - Next Generation Networking and Internet Symposium, Available in IEEE.

Information Centric Networking Research Group(ICNRG),[Online] available at https://irtf.org/icnrg

[49] Stanford University TRIAD project. [Online]. Available: http://gregorio.stanford.edu/triad/

[50] NSA NetInf Project.[Online] Available at http://www.netinf.org/

[51] FP7-GreenICN Project,[Online],Available http://www.greenicn.org/ 